\title{
Studies of Gamma-Glycine Crystals Grown In the Aqueous Solution of Cesium Chloride
}

\author{
T.Gladys Vimala ${ }^{1}$, E.Bena Jothy ${ }^{1}$, P.Selvarajan ${ }^{2}$ \\ ${ }^{I}$ Department of Physics, Women's Christian College, Nagercoil-629151, Tamil Nadu, India. \\ ${ }^{2}$ Department of Physics, Aditanar college of Arts and Science, Tiruchendur-628216,Tamil Nadu,India.
}

\begin{abstract}
Glycine is available in many polymeric forms like $\alpha$-glycine, $\beta$-glycine and $\gamma$-glycine and among these, gamma-glycine ( $\gamma$-glycine) crystallizes in a non-centrosymmetric space group and hence it possesses nonlinear optical $(N L O)$ and piezoelectric properties. Single crystals of gamma-glycine were grown by slow evaporation solution growth technique in the aqueous solution of cesium chloride. To obtain the sample of gamma-glycine, commercially available alpha-glycine and cesium chloride were taken in 2:1 molar ratio and it took about 25 days to harvest crystals of gamma-glycine. The lattice parameters were found by XRD method. The relative SHG efficiency was measured for the sample by Kurtz-Perry method. Hardness parameters were determined at different applied loads. Dielectric behavior of the sample was studied by measuring dielectric constant and loss factor at various frequencies and temperatures.Spectral methods like UV-visible transmittance studies were used to characterize the grown crystal.
\end{abstract}

Keywords: gamma-glycine, single crystal, solution growth, XRD, SHG, spectral method

\section{Introduction}

Glycine is the simplest of amino acids and it functions as a neurotransmitter and one of the principle components of structural proteins, enzymes and hormones. It is available in many forms like alpha-glycine, beta-glycine and gamma-glycine[1,2]. Gamma-glycine crystallizes in a non-centrosymmetric space group and hence it possesses nonlinear optical (NLO) and piezoelectric properties[3]. Gamma-glycine crystals can be grown using many alkali halides like sodium chloride, potassium chloride, lithium chloride, potassium bromide etc. In the normal atmosphere, gamma-glycine exists as a dipolar ion in which carboxyl group is present as a carboxylate ion and amino group is present as ammonium ion. The $\gamma$-form of glycine is thermodynamically the most stable form at room temperature but transforms to the $\alpha$-form at high temperatures. The growth of $\gamma$-form of glycine crystals can be done from aqueous solution or gel in the presence of additives, from supersaturated solution irradiated with plane-polarized laser light, or from surfactant based microemulsion and lamellar phases [4-5]. The polymorphism of glycine was explained that the crystallization of the least stable $\beta$-form and the stable $\gamma$-form $\square \square$ from aquous solution correlated with conditions that had inhibitory effects on the dimer formation [6-9]. In this work, $\gamma$-glycine crystals were grown by solution method using cesium chloride as an additive. The grown crystals have been subjected to many studies like XRD studies, SHG, microhardness studies, dielectric studies and UV-visible spectral studies.

\section{Growth of bulk crystal}

Analar Reagent grade of glycine and cesium chloride were purchased commercially and these chemicals have been taken in the molar ratio of 2:1. The calculated amounts of glycine and cesium chloride were dissolved in double distilled water and stirred well using a magnetic stirrer for about 3 hours. The solution was heated until the synthesized salt of $\gamma$-glycine was obtained. During the synthesis, temperature of the solution was maintained at $50{ }^{\circ} \mathrm{C}$ in order to avoid the oxidation of glycine. Saturated solution was prepared using the synthesized salt of gamma-glycine and water. Then the filtered solution was kept in a growth vessel covered with a porous paper. By slow evaporation of solvent at ambient temperature, single crystals of gammaglycine were obtained. Seed crystals were used to grow big-sized bulk crystal of gamma-glycine.

\subsection{Structural and density measurement}

\section{Results and discussion}

The grown single crystals were subjected by single crystal XRD studies using an ENRAF NONIUS CAD4 diffractometer with $\mathrm{MoK}_{\alpha}$ radiation $(\lambda=0.71073 \AA)$ and the obtained data are are $\mathrm{a}=7.055(2) \AA, \mathrm{b}=$ 7.055(2) $\AA, c=5.518(4) \AA, \alpha=90^{\circ}, \beta=90^{\circ}, \gamma=120^{\circ}$. It is observed that gamma-glycine crystal belongs to hexagonal system, with the number of molecules per unit cell $\mathrm{Z}=3$. 
The floatation method was employed for the precise determination of density of crystals. After mixing the liquids bromoform or carbon tetra chloride and xylene in a suitable proportion in a specific gravity bottle, a small piece of a crystal was immersed in the mixture of the liquids. When the sample was attained in a state of mechanical equilibrium, the density of the crystal would be equal to the density of mixture of liquids. The density was calculated using the relation $\rho=\left(\mathrm{w}_{3}-\mathrm{w}_{1}\right) /\left(\mathrm{w}_{2}-\mathrm{w}_{1}\right)$ where $\mathrm{w}_{1}$ is the weight of the empty specific gravity bottle, $w_{2}$ is the weight of the specific gravity bottle with full of water and $w_{3}$ is the weight of the specific gravity bottle full of the mixture of the solution. The calculated value of density of gamma-glycine crystal by floatation method was $1.604 \mathrm{~g} / \mathrm{cc}$. The density was also calculated from the crystallographic data using the relation $\rho=(M . Z) /(N . V)$ where $M$ is the molecular weight of the material used, $\mathrm{Z}$ is the number of molecules per unit cell, $\mathrm{N}$ is Avogadro's number and $\mathrm{V}$ is the volume of the unit cell. The determined value of density of gamma-glycine using the crystallographic data was $1.582 \mathrm{~g} / \mathrm{cc}$.

\subsection{Measurement of second harmonic generation}

Powder second harmonic generation test offers the possibility of assessing the nonlinearity of the new materials. Kurtz powder method is a useful method for characterizing the materials before going through the long and tedious process of growing large optical quality crystals [10]. The Second Harmonic Generation (SHG) conversion efficiency was tested using a set-up of Kurtz and Perry and it was carried out using Qswitched mode locked Nd:YAG laser with first harmonic output at $1064 \mathrm{~nm}$. The grown crystal of gammaglycine was powdered with uniform particle size of about $200 \mu \mathrm{m}$ using a mortar and pestle and it was packed densely between two transparent glass slides separately. The Nd:YAG laser can be operated in two differentmodes. In the single-shot mode, the laser emits an $8 \mathrm{~ns}$ pulse. In the present study, a multi-shot mode of $8 \mathrm{~ns}$ laser pulse with a spot radius of $1 \mathrm{~mm}$ was used. The fundamental laser beam of $1064 \mathrm{~nm}$ wavelength, $8 \mathrm{~ns}$ pulse with $10 \mathrm{~Hz}$ pulse rate was made to fall normally on the sample cell. The power of the incident beam was measured using a power meter. The filters were used to fundamental and residual lights. The green light was detected by a photomultiplier tube and displayed on a Cathode Ray Oscilloscope. KDP crystal was powdered into identical size as that of the sample and it was used as reference material in the SHG measurement. The measured value of SHG efficiency for gamma-glycine crystal is 1.72 times that of KDP.

\subsection{Measurement of hardness}

Hardness of a material is a measure of resistance offered by a lattice against lattice destruction. Measurement of hardness is a non-destructive testing method to determine the mechanical behaviour of the materials. By definition the indentation hardness is the ratio of the applied load to the surface area generated due to indentation. As the hardness properties are basically related to the crystal structure and the bond strength of the material, microhardness studies have been applied to understand the plasticity of the crystals. Hardness tests are commonly carried out to determine the mechanical strength of materials and it correlates with other mechanical properties like elastic constants and yield stress. The hardness is calculated from the area or the depth of indentation produced. The indenter is made up of a very hard material to prevent its deformation by the test piece, so that it can cover materials over a wide range of hardness. In the Vickers hardness test, an indenter with a square based pyramid is used for which the angle between opposite faces is ideally $136^{\circ}$. From the general definition of hardness, for a given diagonal $d \mathrm{~mm}$ of the square impression formed on the sample and for

the load $P$,

Vickers hardness number, $\mathrm{H}_{\mathrm{v}}=\frac{2 \mathrm{P} \sin (\alpha / 2)}{d^{2}}$

$$
\text { Since } \alpha=136^{\circ}, \quad \mathrm{H}_{\mathrm{v}}=\frac{1.8544 P}{d^{2}} \mathrm{~kg} / \mathrm{mm}^{2}
$$

Among the various types of microhardness measurements, the most common and reliable one is the Vickers microhardness test[11]. It uses a square diamond pyramid whose opposite faces contain an angle of $136^{\circ}$. Microhardness measurements were done using a Vickers microhardness indenter (Leitz Weitzler harness tester). In the present work, the grown crystal was subjected to microhardness test for different loads and indentation time given is $10 \mathrm{~s}$. For each load, several indentations were made and the average diagonal length (d) was used to calculate the microhardness number $\left(\mathrm{H}_{\mathrm{v}}\right)$. The plot between hardness values and the corresponding loads for gamma-glycine crystal is drawn and is presented in the figure 1. From the results of microhardness studies, it is observed that hardness number $\left(\mathrm{H}_{\mathrm{v}}\right)$ increases with load for the sample. This can be explained on the basis of depth of penetration of the indenter. When the load increases, a few surface layers are penetrated initially and then inner surface layers are penetrated by the indenter with increase in the load. The measured hardness is the characteristics of these layers and the increase in the hardness number is due to the overall effect on the surface and inner layers of the sample.

National Conference on Current Advancements in Physics $3^{\text {rd }} \& 4^{\text {th }}$ February 2017 


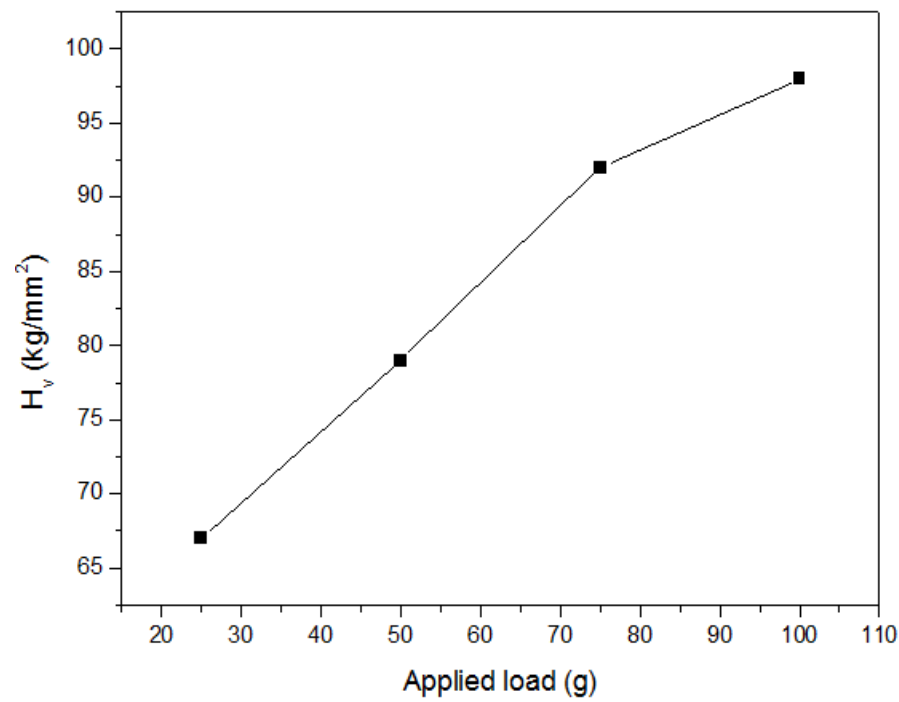

Fig.1: Variation of hardness number with the applied load for gamma-glycine crystal

\subsection{Dielectric studies}

The capacitance and dielectric loss factor $(\tan \delta)$ measurements were carried out using a precision LCR meter (Agilent $4284 \mathrm{~A}$ ) with frequency range of $10^{2}-10^{6} \mathrm{~Hz}$ at room temperature. Dielectric constant $\left(\varepsilon_{\mathrm{r}}\right)$ is related to the symmetry and structure of crystals, the coordination number and the polarizabilities. Various polarization mechanisms in solids such as atomic polarization of the lattice, orientational polarization of dipoles and space charge polarization can be understood very easily by studying the dielectric properties as a function of frequency and temperature for crystalline solids. For the dielectric measurements, the grown crystal was taken and was polished, shaped. The sample was cut into rectangular shapes to the desired thickness of 2-3 mm and polished. For good electrical contact, opposite faces of the sample crystal was coated with good quality graphite.

The variations of dielectric constant $\left(\varepsilon_{\mathrm{r}}\right)$ and dielectric loss with frequency are shown in the figure 2 . . From the measurements, it is observed that the dielectric constant and loss factor of the sample decrease with increase of frequency. The high value of $\varepsilon_{\mathrm{r}}$ at low frequencies may be due to the presence of polarizations namely, space charge, orientational and ionic polarization and its low value at higher frequencies may be due to the loss of significance of these polarizations gradually. The dielectric constant and dielectric loss decrease with increase in frequency and it suggests that the sample seems to contain molecules of varying relaxation times. At higher frequencies, the values of dielectric constant and loss are low because molecules of larger relaxation times may not be able to respond to these higher frequencies[12-13].

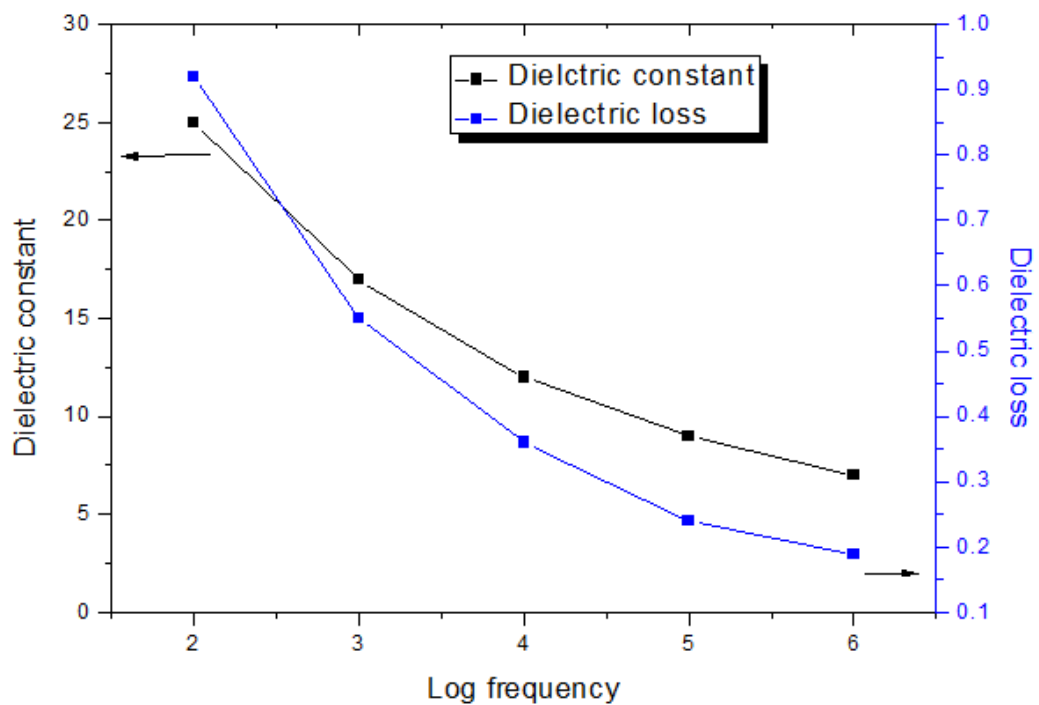

Fig.2: Variations of dielectric constant and dielectric loss as a function of frequency for the grown gammaglycine crystal 
The laser damage in the crystals depends upon the specific properties of material, pulse width, and wavelength of laser used. Laser damage threshold (LDT) studies for the sample were carried out using an Nd:YAG laser (1064 nm, $18 \mathrm{~ns}$ pulse width). The energy of the laser beam was measured by Coherent energy/power meter (Model No. EPM 200). LDT value is determined using the formula $\mathrm{P}=\mathrm{E} / \tau \pi \mathrm{r}^{2}$ where $\tau$ is the pulse width in $n s, E$ is the input energy in $\mathrm{mJ}, \mathrm{r}$ is radius of the spot in mm.[14]. The LDT value is one of the important device related properties of NLO crystals. LDT value is the maximum permissible power that can withstand in a particular crystal. The obtained value of LDT of the grown gamma-glycine crystal is 0.81 $\mathrm{GW} / \mathrm{cm}^{2}$.

\subsection{UV-visible transmission studies}

Transparency is one of the important parameters that a good NLO material should posses for second harmonic generation (SHG). The wider transparency range, the greater will be the practical applicability of that material. The UV-Vis transmission spectrum for the sample is presented in the figure 3 . Low absorption in the entire visible and near infrared region with the low cut-off wavelength at $265 \mathrm{~nm}$ suggests that the material is quite suitable for SHG generation and other related optoelectronic applications. Absorption in the near ultraviolet region arises from electronic transitions associated within the sample. Using the formula $E_{g}=1240 / \lambda(\mathrm{nm})$, the band gap is calculated to be $4.67 \mathrm{eV}$. The good transmission of the crystal in the entire visible region suggests its suitability for second harmonic generation devices.

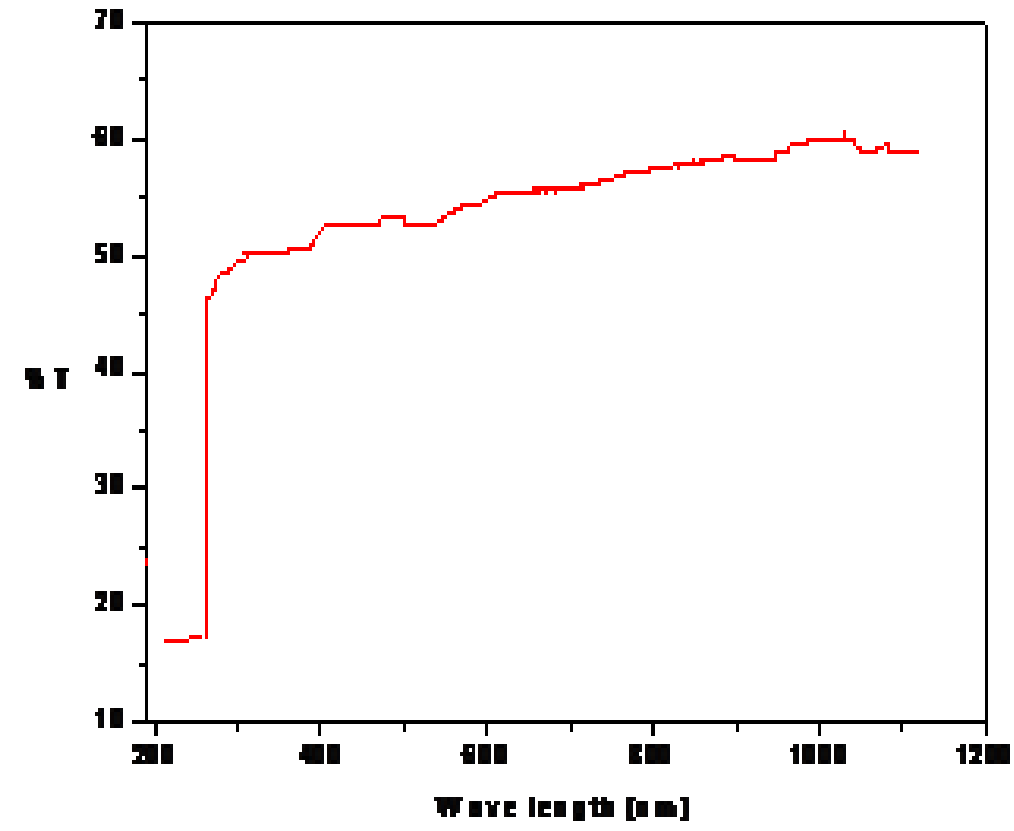

Fig.3: UV-visible transmittance spectrum for gamma-glycine crystal

\section{Conclusion}

Single crystals of gamma-glycine were grown by slow evaporation technique in the aqueous solution of cesium chloride. The XRD studies reveal the hexagonal structure of the sample. The dielectric constant and loss factor were measured for the sample at various frequencies and these values are observed to be decreasing with increase of frequency. Density of the grown crystal was measured by floatation method. The hardness of gamma-glycine crystal is observed to be increasing with the increase of applied load. The SHG and LDT values of the sample were measured. Optical band gap was determined for the grown crystal by UV-visible transmittance spectral method.

\section{Acknowledgements}

The authors are thankful to the management of Women's Christian college, Nagercoil and the management of Adtianar College, Tiruchendur for the encouragement given to us to carry out the research work. The authors are grateful to the research staff members of St.Joseph's College, Trichy, STIC, Cochin University, Cresent Engineering College, Chennai, VIT, Vellore, IIT, Madras for taking the research data to complete this work.

\section{References}


[1] Tapati Mallik, Tanusree Kar, Cryst. Res. Technol. 40 (2005) 778.

[2] R. Muralidharan, R. Mohankumar, P.M. Ushasree, R. Jayavel, P. Ramasamy, J. Crystal Growth 234 (2002) 545.

[3] K. Srinivasan J. Crystal Growth 311 (2008) 156.

[4] J. Zaccaro, J. Matic, A.S. Myerson, B.A. Garetz, Cryst. Growth Des. 1 (2001) 5.

[5] K. Allen, R.J. Davey, E. Ferrari, C. Towler, G.J. Tiddy, Cryst. Growth Des. 2 (2002) 523.

[6] C.S. Towler, R.J. Davey, R.W. Lancaster, C.J. Price, J. Am. Chem. Soc. 126 (2004) 13347.

[7] G.D. Profio, S. Tucci, E. Curcio, E. Drioli, Cryst. Growth Des. 7 (2007) 526.

[8] Y. Iitaka, Acta Crystallogr. 14 (1961) 1.

[9] T.P. Srinivasan a,b, R.IndirajithS a, R.Gopalakrishnan, J. Crystal Growth 318 (2011) 762-767.

[10] S.K.Kurtz, T.T.Perry, J.Appl.Phys. 39 (1968) 3798.

[11] S. Karan, S.P. Sen Gupta, Mater. Sci. and Eng. A357 (2003) 304-307.

[12] K.V. Rao, A.Samakula, J.Appl.Phys. 36 (1965) 2031.

[13] P.Selvarajan, B.N.Das, H.B.Gon , K.V.Rao, J.Mat.Sci. 29(1994)4061.

[14] Redrothu Hanumantharao, S.Kalainathan, Optik, 124 (2013) 2204. 